\title{
Effects of isopropanol on collagen fibrils in new parchment
}

\author{
Lee G Gonzalez ${ }^{1 *}$, Jennifer Hiller ${ }^{2}$, Nick J Terrill ${ }^{2}$, Joanna Parkinson ${ }^{3}$, Kate Thomas $^{1}$ and Tim J Wess ${ }^{1}$
}

\begin{abstract}
Background: Isopropanol is widely used by conservators to relax the creases and folds of parchment artefacts. At present, little is known of the possible side effects of the chemical on parchments main structural componentcollagen. This study uses $\mathrm{X}$-ray Diffraction to investigate the effects of a range of isopropanol concentrations on the dimensions of the nanostructure of the collagen component of new parchment.

Results: It is found in this study that the packing features of the collagen molecules within the collagen fibril are altered by exposure to isopropanol. The results suggest that this chemical treatment can induce a loss of structural water from the collagen within parchment and thus a rearrangement of intermolecular bonding. This study also finds that the effects of isopropanol treatment are permanent to parchment artefacts and cannot be reversed with rehydration using deionised water.

Conclusions: This study has shown that isopropanol induces permanent changes to the packing features of collagen within parchment artefacts and has provided scientific evidence that its use to remove creases and folds on parchment artefacts will cause structural change that may contribute to long-term deterioration of parchment artefacts. This work provides valuable information that informs conservation practitioners regarding the use of isopropanol on parchment artefacts.
\end{abstract}

Keywords: Collagen, Parchment, X-ray Diffraction, Isopropanol, Conservation

\section{Background}

Parchment has been used as a writing material for thousands of years, and many public records and iconic documents are written on the medium. It is long lasting and hardwearing; worldwide there are millions of parchment artefacts held in collections. Parchment is manufactured from the dermis layer of animal skins [1], and its structural matrix is predominantly composed as a composite of the hierarchical protein - collagen, and its denatured form - gelatine [2]. The hierarchy from the molecular to the fibrillar arrangement of collagen is well documented [3]. Three polypeptide chains are arranged into a triple helix to form a collagen molecule. Collagen molecular units, in their native form, are bound in a quarter staggered axial arrangement which subsequently produces a collagen fibril [4].

\footnotetext{
* Correspondence: GonzalezLG@cardiff.ac.uk

'School of Optometry and Vision Sciences, Cardiff University, Maindy Road, Cathays, Cardiff, UK

Full list of author information is available at the end of the article
}

When denaturation (the unfolding of the collagen molecules) occurs, the structural integrity and exquisite hierarchy of molecules, fibrils and fibres is lost. Large quantities of gelatine in parchment documents can be undesirable because it can put documents at risk to physical changes, such as fragmentation [5]. Moreover, gelatine will dissolve easily in water because the intermolecular bonds cannot withstand the stress imposed from osmotic swelling. The structural integrity of gelatine is affected by temperatures $>30^{\circ} \mathrm{C}$ and relative humidity $>70 \%$ and $<30 \%$ RH $[6,7]$.

A challenge for conservation practitioners is to balance the need to preserve historical parchment artefacts and make them accessible to the public. There are number of interventions, (for example laser cleaning and humidification) used by conservation practitioners to help parchment artefacts remain fit for purpose such as to be read, or displayed. In addition, to relax undesirable creases and folds in parchment documents conservation practitioners often use applications of approximately 80:20 isopropanol (IPA):deionised water $\left(\mathrm{dH}_{2} \mathrm{O}\right)$. The application procedure involves applying IPA solutions locally to the crease or 
fold followed by light pressing applied manually [8]. Studies of the interaction between monohydric alcohols, i.e. those with one hydroxy group, such as IPA $\left(\mathrm{CH}_{3}\right.$ $\mathrm{CHOH}-\mathrm{CH}_{3}$ ) and collagen indicate that they incite alteration of the hydrogen bonding network of collagen [9], this may be the basis by which the required flexibility of parchment is induced.

To determine if the use of IPA is detrimental to parchment artefacts it is necessary to understand if IPA incites structural changes within parchments, and further determine whether structural changes are transient during treatment, or permanent post treatment. Because the collagen hierarchical arrangement is highly repetitive, changes in short and long order distances between collagen molecules is an effective way of measuring alteration to the collagen molecular structure. It is possible to investigate structural changes by using X-ray Diffraction (XRD) $[3,10,11]$. Changes in the packing features of collagen such as the distances between collagen molecules in the lateral plane of the collagen fibril (intermolecular lateral packing), the distance between collagen molecules along the fibril (axial D-periodicity) and the distance between amino acids along the polypeptide chain (helical rise per residue) are some of the common features measured using XRD.

In the present work, we have investigated changes to the packing features of the collagen component within parchment in the presence of IPA by use of XRD. Wide Angle $\mathrm{X}$-ray Diffraction (WAXD) was used to capture diffracting $\mathrm{X}$-rays corresponding to the intermolecular lateral packing and the helical rise per residue and Small angle X-ray Diffraction $(\mathrm{SAXD})$ was used to capture the diffracted X-rays corresponding to the axial D-periodicity. We explore changes in the packing features of collagen molecules within collagen fibrils when parchments samples are immersed in IPA and after the samples have been airdried. This procedure was designed to mimic the IPA treatment performed by conservation practitioners.

\section{Results and discussion}

Figure 1 shows an example of a 2D WAXD pattern and the corresponding linear intensity vs scattering vector trace generated from a typical parchment sample. In WAXD patterns the equatorial reflection due to the intermolecular lateral packing arises from the interference function due to the lateral distance between nearest neighbour collagen molecules. The scattering region corresponds to the diffuse scattering of the non-crystalline regions of the collagen fibril and some scatter from the collagen helix; and the meridional reflection due to the helical rise per residue corresponds to the distance between polypeptide subunits [Gly-Y-X] along a polypeptide chain. Figure 2 shows a typical SAXD pattern and the corresponding linear trace generated from a parchment

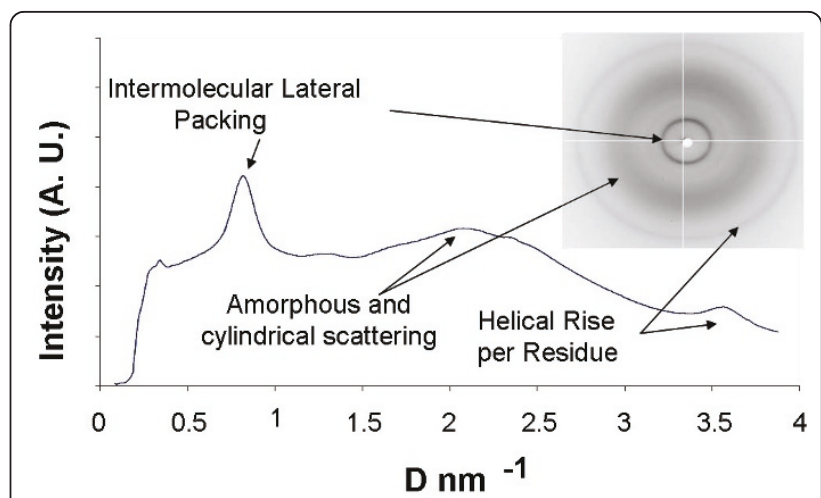

Figure 1 2D WAXD pattern and corresponding 1D linear trace

sample. The axial $\mathrm{D}$ period distribution of electron density along the collagen fibril gives rise to the meridional X-ray scattering observed in a SAXD 2D pattern [12]; in dry parchment the axial D-periodicity is typically around 60$65 \mathrm{~nm}[11,13-15]$.

When immersed in IPA solution it was found that the fibrillar repeat meridional (axial) D-periodicity of the parchment samples ranged between $65.1 \mathrm{~nm}(+/-0.15$ $\mathrm{nm})$ for samples treated with 10-60\% IPA and $65.6 \mathrm{~nm}$ $(+/-0.16 \mathrm{~nm})$ for samples treated with $70-100 \%$ IPA, the control sample (that had been immersed in $\mathrm{dH}_{2} \mathrm{O}$ ) exhibited a repeat of $65.1 \mathrm{~nm}(+/-0.12 \mathrm{~nm})$. Measurement of the intermolecular lateral packing and helical rise per residue when samples were subsequently wetted with $\mathrm{dH}_{2} \mathrm{O}$ was not obtained. The structural information taken from the WAXD and SAXD patterns when the parchment samples had been treated with IPA solutions and air-dried is given in Table 1. After IPA treatment and air-drying regimes, the axial D-periodicity is observed to be shorter in all samples that have been treated with IPA than that found in the control, with the shortest length of the axial periodicity when the concentration of IPA in solution applied was $\geq 80 \%$. This indicates that IPA induces a change in the axial relationship

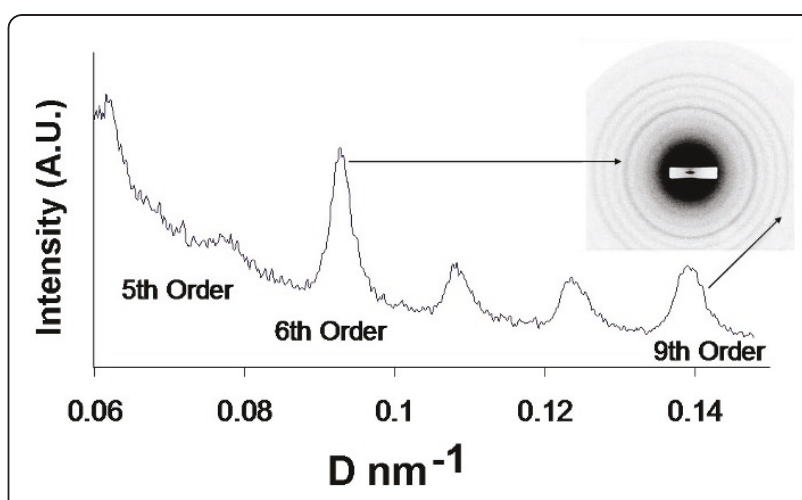

Figure 2 2D SAXD pattern and corresponding 1D linear trace. 


\begin{tabular}{llll}
\hline IPA \% & $\begin{array}{l}\text { Intermolecular lateral } \\
\text { packing } \mathbf{n m}(\mathbf{s})\end{array}$ & axial D-periodicity $\mathbf{n m}(\mathbf{s d})$ & $\begin{array}{l}\text { helical rise per residue } \\
\mathbf{n m}(\mathbf{s d})\end{array}$ \\
\hline 0 & $1.210(+/-0.007)$ & $62.6(+/-0.13)$ & $0.286(+/-<0.0006)$ \\
10 & $1.211(+/-0.012)$ & $62.3(+/-0.00)$ & $0.282(+/-0.0008)$ \\
20 & $1.211(+/-0.009)$ & $62.4(+/-0.14)$ & $0.282(+/-<0.0006)$ \\
30 & $1.203(+/-0.003)$ & $62.3(+/-0.27)$ & $0.282(+/-<0.0009)$ \\
40 & $1.200(+/-<0.008)$ & $62.4(+/-0.21)$ & $0.280(+/-0.0007)$ \\
50 & $1.182(+/-0.007)$ & $62.5(+/-0.16)$ & $0.280(+/-0.0004)$ \\
60 & $1.182(+/-0.006)$ & $62.1(+/-0.16)$ & $0.280(+/-<0.0008)$ \\
70 & $1.169(+/-0.004)$ & $62.1(+/-0.13)$ & $0.279(+/-<0.0001)$ \\
80 & $1.169(+/-0.005)$ & $61.3(+/-0.14)$ & $0.278(+/-0.0005)$ \\
90 & $1.169(+/-<0.007)$ & $61.3(+/-0.06)$ & $0.278(+/-0.0007)$ \\
100 & $1.148(+/-0.005)$ & $61.1(+/-0.14)$ & $0.277(+/-<0.0004)$ \\
\hline
\end{tabular}

between collagen molecules along the fibril axis and that this is concentration dependent. In addition, the intermolecular lateral packing of collagen molecules within the fibril steadily decreases from 1.210 to $1.148 \mathrm{~nm}$ as a function of increasing IPA in the solution used prior to drying. The results also show the helical rise per residue to be smaller when the quantity of IPA in solution $\geq 40 \%$. The helical rise per residue changes are all of a similar order - between 95 and $97 \%$ - as a percentage of the repeating unit that they represent. It should be noted that the change of the axial rise per residue therefore could account for the change in the axial periodicity of the fibrillar repeating unit.

In the second experimental regime samples were immersed in IPA, air dried, then immersed (washed) with $\mathrm{dH}_{2} \mathrm{O}$, and air-dried again; the XRD measurements were then repeated. It is found that from the rehydration experiment that the axial D-periodicity of the collagen molecules and the helical rise per residue increases significantly for those samples treated with 70-100\% IPA solutions. Here the original meridional (axial) fibrillar D periodicity extended from 62.2 to $64.6 \mathrm{~nm}$ and the helical rise per residue increased from $0.286-0.290 \mathrm{~nm}$ as IPA concentration used increased. In contrast the intermolecular lateral packing was found to decrease for samples treated with more concentrated IPA solutions typically 1.212 $\mathrm{nm}$ to $1.132 \mathrm{~nm}$ as IPA concentration increased.

These results show that the packing features of collagen within parchment are altered by exposure to IPA and are non-reversible with rehydration of parchment. Because the intermolecular lateral packing was found to be lower for parchment samples treated with more concentrated IPA solutions, there is an indication that the IPA treatment has removed water molecules from within the collagen fibril; thus inducing a rearrangement of intermolecular bonding which has brought the collagen molecules in the lateral plane of the fibril closer to together. This rearrangement of water populations may also explain the change in the helical rise per residue of the collagen molecule and the concomitant increase of the axial D-periodicity.

The change in the axial D-periodicity and helical rise per residue also indicates that the process of IPA-collagen interaction involves IPA molecules binding to the collagen triple helix and when in sufficient quantity causing distortion of the collagen molecular structure. The IPA-collagen interaction could be from IPA molecules forming complexes with amino acid side chains, either, in a single helix chain between adjacent collagen molecules, or between helix chains within a triple helix. It is also possible that IPA causes a disruption of the amino acid bonding along the collagen triple helix, which subsequently induces rearrangement of intra and intermolecular bonding.

IPA is a methylated derivative of water and can impair the hydrogen bonding ability of a collagen molecule by removing hydrogen bond donors and sterically blocking access to the molecule [16]. Kuznetzova et al [16] showed that IPA molecules can absorb onto the collagen surfaces and suggested that IPA could induce a change in the structural parameters of collagen through changes in the helical winding of the molecules. Moreover, Usha et al [17] reported that IPA molecules could remain permanently bound to a collagen structure. Bonincontra et al [18] and Calandrini et al [19] reported that although the general effect of IPA treatment was destabilisation of the macromolecular structure, the collagen conformation had changed, resulting in alteration of the collagen molecular dimensions. The authors suggested that this was caused by the collagen molecule being wound more tightly as a result of rearrangement of water within the collagen lattice.

Water molecules are thought to be associated with collagen in four different phases, these are: structural water (phase I) thought to bound tightly within the collagen triple helix either through a double or triple 
hydrogen bond. Bound water (phase II), where water molecules form bridges between neighbouring polypeptide chains and act as receptors for $\mathrm{CH}-\mathrm{O}$ hydrogen bonds [20]. Free water (phase III) where water is fixed by one hydrogen bond between polypeptide chains or fixed in the hole zones at the end of the polypeptide chain, and finally transition water (phase IV), where water is bound between the fibrils [21,22]. Although comparatively dry, collagen based materials will often contain significant levels of water and the majority is held at a molecular level; which is believed to have a stabilising effect through participation in interchain bonding $[23,24]$.

The results show that within parchment, IPA solutions bring about a disruption of the collagen molecular unit. Parchment samples treated with IPA solutions above 30\% and air-dried exhibit a reduction in the structural dimensions of the collagen fibrils indicating that IPA has perturbed the collagen lattice (Table 1). The greatest structural change induced from exposure of the samples to IPA was observed at higher IPA concentrations. The axial D-periodicity and helical rise per residue of the collagen fibrils after the rehydration experiment were observed to return to the original values for those samples exposed to 10-60\% IPA (Table 2). This indicates that at a concentration of $10-60 \%$. IPA only disrupts intermolecular bonding. This is confirmed by the fact that the intermolecular lateral packing distance is not observed to return to its original value. For samples treated with IPA concentration $>70 \%$, none of the structural dimensions measured return to their original values indicating that at higher IPA concentrations there is disruption of the collagen intramolecular bonding as well as the intermolecular bonding. Given the chemical nature of IPA, it is highly likely that the intramolecular disruption observed is caused by displacement of structural water (Phase I and II) within the collagen molecule. Figure 3 shows a

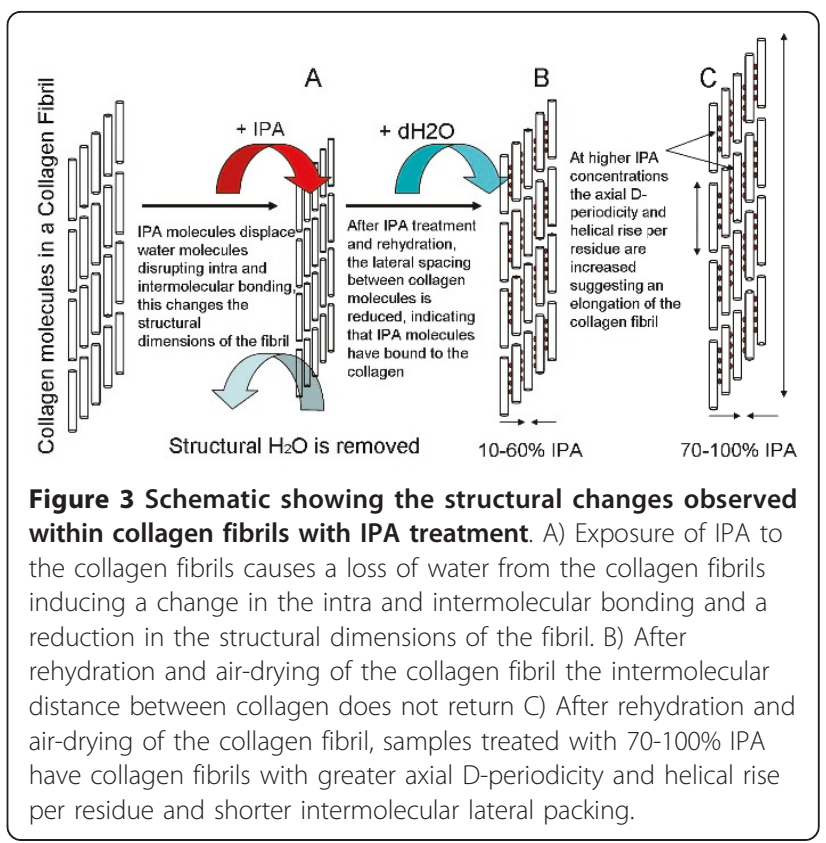

schematic of collagen molecules within a fibril and demonstrates how the dimensions of the fibril change with IPA interaction.

The XRD studies have revealed that IPA molecules interact with collagen molecules within collagen fibrils and permanently disrupt the packing features. The results indicate that the process of IPA-collagen interaction involves a rearrangement of water, and, intra and intermolecular bonding; the use of IPA can therefore be considered as a chemical that causes adverse structural changes to parchment artefacts. The IPA induced collagen molecular structural change will undoubtedly make the collagen component within parchment more susceptible to gelatinisation (the process of transforming a solid structure into a gel), however; the potential for

Table 2 Structural information obtained from XRD patterns of collagen fibrils after IPA treatment, rehydration and airdrying

\begin{tabular}{cccc}
\hline IPA \% & $\begin{array}{c}\text { intermolecular lateral packing } \mathbf{n m} \\
(\mathbf{s d})\end{array}$ & $\begin{array}{c}\text { axial D-periodicity } \mathbf{n m} \\
(\mathbf{s d})\end{array}$ & $\begin{array}{c}\text { helical rise per residue } \mathrm{nm} \\
(\mathbf{s d})\end{array}$ \\
\hline 0 & $1.212(+/-0.011)$ & $62.6(+/-0.03)$ & $0.286(+/-0.0007)$ \\
10 & $1.197(+/-<0.001)$ & $62.5(+/-0.06)$ & $0.286(+/-0.0003)$ \\
20 & $1.203(+/-0.006)$ & $62.7(+/-0.02)$ & $0.286(+/-0.0004)$ \\
30 & $1.211(+/-0.004)$ & $62.8(+/-0.03)$ & $0.286(+/-0.0006)$ \\
40 & $1.179(+/-0.004)$ & $62.6(+/-0.09)$ & $0.285(+/-0.0004)$ \\
50 & $1.168(+/-0.004)$ & $62.6(<0.07)$ & $0.286(+/-0.0001)$ \\
60 & $1.176(+/-0.002)$ & $62.5(+/-0.08)$ & $0.285(+/-0.0005)$ \\
70 & $1.146(+/-0.002)$ & $63.7(+/-0.04)$ & $0.289(+/-<0.0001)$ \\
80 & $1.149(+/-0.003)$ & $63.7(<0.01)$ & $0.289(+/-0.0009)$ \\
10 & $1.151(+/-0.004)$ & $64.0(+/-0.05)$ & $0.289(+/-0.0004)$ \\
\hline
\end{tabular}


gelatinisation to occur post IPA treatment is unclear from these results.

This study has been useful for identifying the physicochemical basis of which IPA induces changes to the structure of parchment artefacts. It has also shown that the structural changes are concentration dependent, where higher IPA concentrations bring about permanent IPA-collagen bonding. To understand further the impact of IPA on parchment, additional XRD investigations could be performed that analysed the change in proportions of collagen to gelatine in parchment with IPA treatment, and this could be carried out on parchment artefacts with a range of conditions i.e. highly damaged, damaged etc. It would also be useful to understand how the conformation of the collagen molecular structure is altered with IPA treatment and this could be analysed using Fourier Transform Infrared spectroscopy.

\section{Experimental \\ Materials}

Samples used in this study were obtained from a newly manufactured parchment sheet sourced from W Cowley Parchment \& Vellum, Newport Pagnell, UK. Newly manufactured and historic parchments frequently differ in their relative proportions of collagen and gelatine, where historic parchment documents commonly contain high quantities of gelatine [13]. Since the collagen hierarchical structure of newly manufactured parchment is the same as that found in historic parchment records, the results are therefore of relevance to all parchment records regardless of age. All samples used were taken from the same area of the parchment sheet and cut to have the same dimensions. This was done for the purpose of minimising the variation in the orientation of the collagen fibres between the samples used.

Previous Microfocus X-ray scattering experiments have shown that IPA solutions can alter the structure of parchment when it is applied to parchment surfaces [25]. This work expands upon these findings by addressing the physicochemical basis by which the intervention of IPA treatment causes parchment structural changes. The application of IPA solutions to the surfaces of parchments is not a standardised practise; therefore for constituency, it is necessary to immerse the entire parchment sample in IPA solution. The IPA treatment involved the complete immersion of the samples in IPA solution for 24 hours. In the following procedures, parchment samples were measured while immersed in IPA solution and after air-drying. Three XRD measurements were made at different points on each parchment sample and the structural dimensions reported are the average values determined from these measurements.

All chemicals used in this study were sourced from Sigma, Poole, UK. Isopropyl alcohol used was of HPLC grade, concentration - $1 \mathrm{M}$. $\mathrm{dH}_{2} \mathrm{O}$ (Millipore - $18 \mathrm{M}^{\prime} \Omega$ ) was used for all experiments. The samples were treated with IPA: $\mathrm{dH}_{2} \mathrm{O}$ solutions of either 0:100 (control), 10:90, 20:80, 30:70, 40:60, 50:50, 60:40, 70:30, 80:20, 90:10 or $100: 0 \%$, then air-dried for 1 day, rehydrated by saturating with $\mathrm{dH}_{2} \mathrm{O}$ and finally air-dried for 1 day.

\section{X-Ray diffraction measurements}

WAXD patterns were collected on the PX and Fibre Diffraction beamline (14.1) at Synchrotron Radiation source, Daresbury Laboratory, Daresbury, UK. Diffraction patterns were generated from $\mathrm{X}$-rays having a wavelength $1.55 \AA$. The sample to detector distance was $140 \mathrm{~mm}$. The exposure time was $40 \mathrm{~s}$ per sample repetition. Diffraction patterns were collected using a charge coupled (CCD) X-ray Quantum 4 ADSC detector (ADSC, Poway, CA). The position of the diffraction orders in respect to one another and a calibration of a known standard (Calcite) were used to determine the position of the reflection due to the intermolecular lateral packing and the helical rise to residue.

SAXD patterns were collected on the non-crystalline diffraction beamline (I22) at the Diamond Light Source, Oxfordshire, UK. 2D Diffraction patterns were generated from X-rays with a wavelength of $1.12 \AA$. The sample to detector distance used was $6 \mathrm{~m}$; thus permitting the meridional series of reflections to be collected. The exposure time was $20 \mathrm{~s}$ per sample repetition. Diffraction patterns were collected using a RAPID 2 dimensional gas filled electronic detector system [26] The data files obtained comprised $1024 \times 1024$ pixel elements [27]. The positions of the diffraction orders in respect to one another and a calibration of a known standard (Silver Behenate) were used to measure the axial D-periodicity of the samples.

WAXD and SAXD patterns were collected 1) after the IPA immersive treatment followed by air-drying and 2) after immersion in IPA followed by rehydration with water and then followed by air-drying. All samples were placed into the X-ray beam and held in place in a purpose-built brass vessel. Throughout the experiment, the transmission of X-rays through the parchment samples occurred from the grain to the flesh side (a transmission thickness of typically 300 microns). The centre of diffraction was defined, and all data files were converted from 2D diffractograms to $1 \mathrm{D}$ linear intensity profiles vs scattering angle using FibreFIX software [28]. The linear intensity profiles were plotted using Microsoft Excel ${ }^{\mathrm{TM}}$. Peakfit4 (AISL software), a 1D peak fitting program, was used to determine the peak size shapes and integrated intensity of the linear profiles. From the position of the maxima of the reflections, the real lattice space values that represent characteristic structural dimensions of a collagen fibril are obtained using Bragg's equation. 


\section{Conclusions}

Analysis of parchment treated with IPA solutions has shown that IPA induces a permanent change in the packing features of collagen within parchment. This means that the change caused to the parchment structure is significant to the integrity of the parchment artefact. For conservation practitioners, these findings suggest that the use of IPA to relax creases and folds is damaging to the collagen component of the parchment record. It is recognised in this study that saturating parchment samples with IPA solution does not represent the brushing technique used by conservators; however, it shows that the collagen component of parchment is affected by IPA treatment.

\section{Abbreviations \\ 1D: 1 dimensional; 2D: 2 dimensional; WAXD: Wide Angle X-ray Diffraction; SAXD: Small Angle X-ray Diffraction; IPA: Isopropanol; dH2O: Deionised water; s: Second; nm: Nanometre; mm: Millimetre; m: Metre; $\AA$ : Angstrom; M' $\Omega$ : Mega Ohm; M: Molar; Gly: Glycine; RH: Relative Humidity; HPLC: High performance liquid chromatography.}

\section{Acknowledgements}

This research was supported by funding received from the National Archives of Scotland and The National Archives. The authors wish to thank Linda Ramsay and the conservation team of NAS, Nancy Bell and the conservation team at TNA, and Dr Marc Malfois and for their contributions to this work.

\section{Author details}

'School of Optometry and Vision Sciences, Cardiff University, Maindy Road, Cathays, Cardiff, UK. ²Diamond Light Source Ltd, Diamond House, Harwell Science and Innovation Campus, Didcot, Oxfordshire. ${ }^{3}$ AstraZeneca, Safety Division, Alderley Park, Macclesfield, Cheshire, UK.

\section{Authors' contributions}

LG and TJW conceived the study, participated in the design and coordination of the experiments and data interpretation and helped draft the manuscript. JH and NJT designed and built the experimental equipment and contributed to the acquisition of the data. JG and KT produced samples, carried out acquisition of data, performed data analysis and data interpretation. All authors read and approved the final manuscript.

\section{Competing interests}

The authors declare that they have no competing interests.

Received: 16 December 2011 Accepted: 31 March 2012

Published: 31 March 2012

\section{References}

1. Poole JB, Reed R: The preparation of leather and parchment by the dead Sea scrolls community. Technol Culture 1962, 3:1-26.

2. Kennedy CJ, Wess TJ: The structure of collagen within parchment - a review. Restaurator Int J Preservation Library Archival Mater 2003, 24:61-80.

3. Maxwell CA, Wess TJ, Kennedy CJ: X-ray diffraction study into the effects of liming on the structure of collagen. Biomacromolecules 2006, 7:2321-2326.

4. In Recent studies with the electron microscope on ordered aggregates of the tropocollagen molecule. Edited by: Hodge AJ, Petruska JA. London: London Academic Press; 1963:

5. Bigi A, Panzavolta S, Rubini K: Relationship between triple-helix content and mechanical properties of gelatin films. Biomaterials 2004, 25:5675-5680.

6. Bancroft WD: The action of water vapour on gelatine. J Phys Chemi 1912, 16:395-406.

7. Kontny M, Mulski CA: Gelatin capsule brittleness as a function of relative humidity at room temperature. Int J Pharamaceutics 1989, 54:79-85.
8. Vinas V: The Use of polyethylene Glycol in the Restoration of Parchment London: Butterworths; 1987.

9. Usha R, Ramasami T: Influence of hydrogen bond, hydrophobic and electrovalent salt linkages on the transition temperature, enthalpy and activation energy in rat tail tendon (RTT) collagen fibre. Thermochimica Acta 1999, 338:17-25.

10. Wess TJ, Drakopoulos M, Snigirev A, Wouters J, Paris O, Fratzl P, Collins M, Hillier J, Nielsen K: The use of small-angle X-Ray diffraction studies for the analysis of structural features in archaeological samples. Archaeometry 2001, 43:117-129.

11. Wess TJ, Nielsen K: Analysis of Collagen Structure in Parchment by Small Angle X-ray Diffraction London: Archetype Publications; 2002.

12. Burge RE, Randall JT: The equivalence of electron-microscope and X-ray observations on collagen fibres. Proceedings of the Royal Society A 1955, 233:1-16.

13. Kennedy CJ, Hiller JC, Lammie D, Drakopoulos M, Vest M, Cooper M, Adderley WP, Wess TJ: Microfocus X-ray diffraction of historical parchment reveals variations in structural features through parchment cross sections. Nano Letters 2004, 4:1373-1380.

14. Kennedy CJ, Vest M, Cooper M, Wess TJ: Laser cleaning of parchment: structural, thermal and biochemical studies into the effect of wavelength and fluence. Applied Surface Science 2004, 227:151-163.

15. Fessas D, Signorellia M, Schiraldia A, Kennedy CJ, Wess TJ, Hassel B, Nielsen K: Thermal analysis on parchments I: DSC and TGA combined approach for heat damage assessment. Thermochimica Acta 2006, 447:30-35.

16. Kuznetsova N, Rau DC, Parsegian VA, Leikin S: Solvent hydrogen-bond network in protein self-assemble: solvation of collagen triple helices in nonaqueous solvents. Biophysical J 1997, 72:353-362.

17. Usha R, Maheshwari R, Dhathathreyan A, Ramasami T: Structural influence of mono and polyhydric alcohols on the stabilization of collagen. Colloids Surf B Biointerfaces 2006, 48:101-105.

18. Bonincontro A, De Francesco A, Matzeu M, Onori G, Santucci A: Conformational changes of lysozyme in water-ethanol mixtures. Colloids Surf B Biointerfaces 1997, 10:105-111.

19. Calandrini V, Fioretto D, Onori G, Santucci A: Role of hydrophobic interactions on the stabilisation of native state of globular proteins. Chem Physics Lett 2000, 324:344-348.

20. Ramachandran GX, Chandrasekharan R: Interchain hydrogen bonds via bound water molecules in the collagen triple helix. Biopolymers 1968, 6:1649-1658

21. Pineri $\mathrm{MH}$, Escoubes $M$, Roche G: Water-collagen interactions: calorimetric and mechanical experiments. Biopolymers 1978, 17:2799-2815.

22. Zhang D, Chippada U, Jordan K: Effect of the structural water on the mechanical properties of collagen-like microfibrils: a molecular dynamics study. Annals Biomedical Eng 2007, 35:1216-1230.

23. Budrugeac $P$, Trandafir $V$, Albu M: The effect of the hydration degree on the hydrothermal and thermo-oxidative stability of some collageneous matrices. J Thermal Anal Calorimetry 2003, 72:581-585.

24. Bella J, Brodsky B, Berman HM: Hydration structure of a collagen peptide. Structure 1995, 3:893-906.

25. Kennedy CJ, Hiller J, Maxwell CA, Wess TJ: X-ray Diffraction Analysis of Parchment-Applications to manufacture, conservation and restoration London: James \& James; 2005.

26. Lewis RA, Helsby WI, Jones AO, Hall CJ, Parker B, Sheldon J, Clifford P, Hillen M, Sumner I, Fore NS, et al: The "RAPID" high rate large area X-ray detector system. Nucl Instrum Meth Phys Res Sec A Accelerators Spectrometers, Detectors Assoc Equip 1997, 392:32-41.

27. Towns-Andrews E, Berry A, Bordas J, Mant GR, Murray PK, Roberts K, Sumner I, Worgan JS, Lewis R, Gabriel A: Time resolved x-ray diffraction station: X-ray optics, detectors, and data acquisition. Reviews of Scientific Instruments 1989, 60:2346-2349.

28. Wess TJ, Wess L, Hocking PM: The structure of avian cartilage: a combined X-ray and biochemical analysis. J Comp Pathol 1997, 116:145-155.

doi:10.1186/1752-153X-6-24

Cite this article as: Gonzalez et al: Effects of isopropanol on collagen fibrils in new parchment. Chemistry Central Journal 2012 6:24. 